\title{
Meta
}

Journal des traducteurs

Translators' Journal

\section{RICCARDI, A. (2003) : Dalla traduzione all'interpretazione, Studi d'interpretazione simultanea, Milan, Edizioni Universitarie di Lettere Economia Diritto, 302 p.}

\section{Christian Balliu}

Volume 49, numéro 4, décembre 2004

URI : https://id.erudit.org/iderudit/009794ar

DOI : https://doi.org/10.7202/009794ar

Aller au sommaire du numéro

Éditeur(s)

Les Presses de l'Université de Montréal

ISSN

0026-0452 (imprimé)

1492-1421 (numérique)

Découvrir la revue

Citer ce compte rendu

Balliu, C. (2004). Compte rendu de [RICCARDI, A. (2003) : Dalla traduzione

all'interpretazione, Studi d'interpretazione simultanea, Milan, Edizioni

Universitarie di Lettere Economia Diritto, 302 p.] Meta, 49(4), 935-938.

https://doi.org/10.7202/009794ar d'utilisation que vous pouvez consulter en ligne.

https://apropos.erudit.org/fr/usagers/politique-dutilisation/ 
original Esperanto (pp. 25-27). Its unsigned English translation, also from Prague on the same date, figures on pages 29-31. In addition, Ngùgì Wa Thiong'o's "Imperialism of Language," drawn from his English, a Language for the World? appears in a translation from the Gikùyù, by the author and Wangùi wa Goro, on pages 169-181.

As well, the excerpt from Elias Canetti's The Play of the Eyes (pp. 301-307) was translated from the German by Ralph Manheim for the publication of Canetti's memoirs in English in 1999. Marjorie Agosín's contribution, from “Words: A Basket of Love” (pp. 321326), a chapter of this author's The Alphabet in My Hands: A Writing Life, was translated from the Spanish by Nancy Abraham Hall. Hana Wirth-Nesher translated Baal-Makhshoves' "One Literature in Two Languages" (pp. 99-109) from the Yiddish, the other language alluded to being Hebrew. Finally, the editor himself interviewed Ha Jin (pp. 81-84) and Chang-rae Lee (pp. 91-94), in English, and it is the transcript of each of these interviews which appears here. In the case of all the 19 other texts, their originally non-Anglophone authors wrote them themselves, in English.

Writers as well known as Salman Rushdie ("Damme, This Is the Oriental Scene for You!," pp. 245-260), Arthur Koestler (an excerpt from “Becoming Anglicized,” pp. 291-292), Chinua Achebe ("The African Writer and the English Language," pp. 191-200), Ian Buruma (“The Road to Babel," pp. 9-22), and Ilan Stavans (“Autobiographical Essay," pp. 113-127) are represented here. The longest and most engaging piece, however, is Luc Sante's "Dummy" (pp. 141-161), whose author is an American of Francophone Belgian origin, well known especially for his articles in The New York Review of Books.

Sante's text starts with an amusing account of how English, French, and Walloon feel in his head, and the visual, auditory, and olfactory images he associates with each of these languages. It continues, though, with a detailed account of the external history and macrosociolinguistics of the relation between Walloon and French. We learn, for example (pp. 153-154), that the "golden age" of Walloon literature was brief, starting about 1880, peaking exactly a century ago, and ending even before World War I. According to the author, it is not only 20th-century life that killed Walloon, but the French attitude toward language, which stigmatizes social and regional variation. He claims that the general Belgian reaction to this attitude has been uncritical acceptance (pp. 149-150), which has led not only to the loss of the indigenous Romance language of the peasant and working classes (Walloon), but also, and more humiliatingly, to that of "Belgicisms" within French. He even blames the Academy's attempts to suppress linguistic changes for the loss of the latter language's international political power to English (p. 150), an opinion sometimes voiced by Francophone linguists as well.

In concluding, Sante returns to the "spaces" occupied by his languages in his mental universe, epitomizing the sentiments of several multilingual authors represented here (p. 161):

I don't have a house, only this succession of rented rooms. That sometimes makes me feel as though I have no language at all, but it also gives me the advantage of mobility. I can leave, anytime, and not be found.

Kathleen Connors University of Montreal, Montreal, Canada

\section{Riccardi, A. (2003): Dalla traduzione all'interpretazione, Studi d'interpretazione} simultanea, Milan, Edizioni Universitarie di Lettere Economia Diritto, 302 p.

Alessandra Riccardi, professeur associé d'interprétation simultanée à la Scuola Superiore di Lingue Moderne per Interpreti e Traduttori de l'Université de Trieste, est interprète de conférence et rassemble dans ce livre plusieurs études sur l'interprétation, et plus particulièrement la 
simultanée, fruit de son expérience professionnelle. Il s'agit en réalité d'une monographie qui tente de faire l'état de la question, en prenant en compte tant les aspects théoriques que pratiques ou historiques. Le lecteur se voit ainsi offrir un vaste panorama critique des recherches dans le domaine de l'interprétation, sans oublier le domaine professionnel.

Le chapitre introductif intitulé Traduzione e interpretazione définit les rôles respectifs de la traduction et de l'interprétation par une plongée dans l'histoire. La dimension diachronique permet d'examiner l'évolution historique du sens attribué au vocable latin interpretari, lequel, aux yeux de l'auteur, a servi à désigner tantôt la traduction, tantôt l'interprétation, tantôt les deux, à partir de l'époque romaine. À la différence des traductions littéraire et philosophique, l'interprétation aurait joui dans le passé d'un prestige moindre, ce qui expliquerait qu'elle ait été négligée par les chercheurs. En réalité, nous n’avons pas de preuve de cette allégation et l'histoire semble plutôt montrer que la traduction utilitaire (la pierre de Rosette en est un bon exemple) a occupé pendant de nombreux siècles le devant de la scène.

Quoi qu'il en soit, la perspective diachronique autorise un balisage des notions et une relativisation des acquis de notre époque. La multiplicité des points de vue qui ont jalonné les époques met en évidence la fragilité d'opinions en apparence définitives. L'auteur estime que, si l'interprétation a eu pour objectif et raison d'être la médiation linguistique à des fins militaires, politiques ou commerciales, la traduction a poursuivi des finalités diverses, comme la connaissance de la culture littéraire, philosophique et religieuse d'autres pays. De même, la traduction aurait été pendant longtemps un exercice d'érudition permettant de mettre à l'épreuve ses propres connaissances et aptitudes littéraires et linguistiques. Ici encore, l'affirmation me semble péremptoire et en tout cas inapplicable à l'histoire en général. On sait par exemple que Descartes écrivit d'abord ses Méditations métaphysiques en latin pour obtenir la reconnaissance de ses pairs, puis les donna en français pour s'assurer un public. Une auto-traduction à des fins spécifiques.

Ce premier chapitre s'attarde aussi sur la distinction devenue classique entre traduction littérale et traduction libre, vue à la loupe de l'histoire. On peut y lire que la traduction des textes sacrés privilégiait l'option ad litteram, contrairement à la traduction considérée comme un exercice littéraire et par conséquent ad sensum; qu'à l'époque romaine, la traduction libre était recommandée; que le $\mathrm{XvI}^{\mathrm{e}}$ siècle français (!) a donné naissance au courant des belles infidèles. Au risque de me répéter, outre les erreurs mentionnées, l'histoire de la traduction est beaucoup plus complexe et ne se laisse pas réduire à des considérations aussi génériques, pour des raisons de commodité articulatoire.

L'opposition entre traduction littérale et traduction libre est ensuite illustrée de manière beaucoup plus approfondie par la position de Schleiermacher en la matière, ce qui s'explique par le fait que l'allemand est, aux côtés de l'italien, la langue de travail de l'auteur. Il y a ici un déséquilibre entre le développement consacré à l'histoire en général et la place accordée au seul philosophe allemand, même si bien entendu son rôle aux côtés de Von Humboldt dans la fondation de l'Université de Berlin en 1810 et dans la Bildung elle-même est essentiel.

Le deuxième chapitre, Studi di traduzione e d'interpretazione, passe en revue les positions des théoriciens de la traduction à propos de l'interprétation, confinées il est vrai aux études de la Translationswissenschaft, dont la théorie du Skopos, et des Translation studies, dont Catford, Nida et Newmark, après 1960. Malheureusement, ces orientations ne sont traitées qu'en une dizaine de pages, ce qui ne permet pas de donner une compétence traductologique d'ensemble. Je regrette pour ma part que la traductologie d'expression française, avec des figures de proue comme Vinay et Darbelnet, Mounin, Cary ou Ladmiral pour ne citer qu'eux, n'ait pas reçu le traitement qu'elle méritait assurément. Ces noms, dont plusieurs sont fondateurs, ne figurent pas non plus dans la bibliographie pourtant abondante que l'on trouve en fin de volume. 
Ensuite, les réflexions des spécialistes de la traduction sur l'interprétation sont examinées, avec un éclairage particulier des raisons qui conduisent nombre de théoriciens à englober la problématique de l'interprétation dans la traductologie. La théorie interprétative de la traduction - la théorie du sens - de Seleskovitch revendique non seulement le droit de l'interprétation à sa propre théorisation, ce que Riccardi signale à juste titre, mais encore sa capacité à éclairer les problèmes apparemment spécifiques à la traduction écrite. La dimension communicative de l'acte d'interprétation, les compléments cognitifs et affectifs plus tangibles qu'à l'écrit lui assurent un avantage indéniable dans la quête du sens. Le problème définitoire réside selon moi dans l'équilibre délicat des convergences et divergences qui caractérisent les deux disciplines. D’autres références sont faites à Gile, Salevsky, Pöchhacker et Kalina, brossant ainsi un tableau sommaire de ces 20 dernières années. Le chapitre se termine par une catégorisation typologique de la traduction et de l'interprétation qui reprend, entre autres, l'objet et les modalités de l'activité, ses conditions spatio-temporelles et son mode de réception.

Le troisième chapitre, La dimensione orale dell'interpretazione, approfondit en quelque sorte les leçons du chapitre précédent. Il s'ouvre par une analyse de l'interprétation interlinguistique et de sa fonction communicative, à travers différents paramètres comme l'orateur, la typologie textuelle, l'argument et la langue de travail. Suit un développement sur l'évolution de l'interprétation, notamment de conférence, et le passage de témoin entre la consécutive et la simultanée. Plusieurs jalons sont mentionnés: la conférence de Paix de Paris en 1919 et les tâtonnements des «premiers » interprètes ${ }^{1}$, l'interprétation consécutive à la SDN et l'essor de la simultanée après la Seconde Guerre mondiale.

Ce passage aurait gagné à être approfondi, dans la mesure où une vraie histoire de l'interprétation au $\mathrm{xx}^{\mathrm{e}}$ siècle reste à faire. Même Seleskovitch, qui a cependant beaucoup écrit en matière de théorie, n'a laissé que peu de choses sur sa carrière professionnelle. On sait que les interprètes sont des "gens de l'oral» et que le secret professionnel qui leur est imposé ne laisse que peu de place à la publication de souvenirs. Comme elle, d'autres interprètes (les frères Kaminker, Edmond Cary ou Léon Dostert) qui ont marqué le $\mathrm{xx}^{\mathrm{e}}$ siècle ont disparu sans avoir vraiment consigné ce qui aurait dû faire partie du patrimoine historique de l'interprétation ${ }^{2}$.

Après les deux voies royales de l'interprétation, une attention est accordée aux autres formes, moins prestigieuses mais tout aussi estimables, telles l'interprétation de liaison et le chuchotage. L'interprétation à distance (télé- et vidéoconférence), l'interprétation en langue des signes et pour les médias ne sont pas oubliées. Enfin, le relais et le retour, appelés à mon sens à se développer plus encore dans les prochaines années, font l'objet d'un bref détour à la fin du chapitre. Il s'agit en l'occurrence davantage d'une mention fugitive que d'une véritable étude de ces techniques.

Le quatrième chapitre, Gli studi d'interpretazione, nous fait entrer dans le vif du sujet. Il présente un éventail des recherches sur l'interprétation, en commençant par les premières études psychocognitives et psycholinguistiques dans le domaine de l'interprétation simultanée. Il s'agissait de suivre en temps réel les mécanismes à l'œuvre dans la production et la réception du message. Le stockage, le traitement et l'utilisation de l'information par l'interprète faisaient l'objet d'une attention toute particulière, sans oublier la segmentation du discours, le décalage ou la division de l'attention entre la compréhension du texte de départ et la reformulation du texte d'arrivée. Il faut dire que depuis les années 1970 des progrès notables ont été enregistrés, sans que nous ne sachions encore aujourd'hui ce qui se passe réellement dans la «boîte noire» de l'interprète.

Riccardi considère à raison que l'ouvrage de Seleskovitch L'interprète dans les conférences internationales: problèmes de langage et de communication (1968), est un livre fondateur; cette étude, comme les autres qui seront publiées dans son sillage par elle-même et Marianne Lederer, établira la théorie $d u$ sens qui, sans doute aucun, donnera à l'interprétation ses lettres 
de noblesse théoriques. On y retrouve à mon avis le modèle tripartite de Nida - analyse, transfert, restructuration - publié quatre ans auparavant ${ }^{3}$ et une volonté d'asseoir l'interprétation dans le giron universitaire.

La relation entre sciences cognitives et interprétation est illustrée par plusieurs modèles: le modèle de Massaro, le modèle de Moser et le modèle de répartition d'efforts de Gile. Les recherches en neuropsychologie et en neurolinguistique se focalisent notamment sur le bilinguisme et le rôle de la mémoire en interprétation simultanée. De même, l'auteur prend en considération les tests d'aptitude à l'interprétation ainsi que les exercices de propédeutique, pour conclure avec les développements les plus récents en la matière et la mise au point de critères de qualité satisfaisants au plan méthodologique.

C'est précisément ce dernier point, la qualité, problème encore entier, qui est au cœur du cinquième et dernier chapitre, Qualità. La qualité est à mon sentiment la question cruciale de l'interprétation aujourd'hui et la définition de cette notion a des implications évidentes tant sur la formation que sur la pédagogie du marché. Il est vrai, comme l'affirmait Seleskovitch, qu' «un interprète médiocre ne sert à rien». En l'absence de critères de qualité qui fassent l'unanimité, je me range volontiers à l'opinion de Gentile: «invariably and inexorably the tendency will be to concentrate on the evaluation of language skills, in part because it is easier to justify one's assessment in terms of language than in terms of communication ${ }^{4} »$.

L'analyse des critères de qualité se fonde sur des variables comme le respect du contenu et la fonction de communication, et fait le départ entre l'interprétation directe pour le public et le relais qui obéit évidemment à d'autres impératifs. Au bout devrait se trouver la notion d'équivalence, ce casse-tête traductologique qui ne cesse de hanter les esprits depuis de nombreux siècles. Bien entendu, l'ambition de l'auteur n'est pas de résoudre le problème, mais de baliser les acquis récents et d'ouvrir des pistes prometteuses. J'aurais néanmoins souhaité que la réalité quotidienne du marché soit plus longuement évoquée.

Le livre s'achève par une bibliographie très abondante et particulièrement fouillée. Le lecteur candide comme l'interprète professionnel y trouveront nombre de références intéressantes et un point de départ utile pour d'éventuelles recherches.

Christian Balliu

Haute École de Bruxelles, Bruxelles, Belgique

\section{NOTES}

1. Voir Jean Herbert: «How conference interpretation grew», in David Gerver and H. Sinaiko (éd.), Language Interpretation and Communication, New York/Londres, Plenum Press, 1978, p. 6 et suivantes.

2. Il y a heureusement des exceptions, par exemple: les Mémoires d'Eugen Dollmann (1963), l'interprète des tête-à-tête entre Hitler et Mussolini, ou le Silent Missions de Vernon Walters (1978), l'interprète de Truman, Eisenhower, Nixon et Kissinger.

3. Eugene A. Nida, Toward a Science of Translating, Leyde, E.-J. Brill, 1964.

4. Adolfo Gentile, «Community interpreting or not?», in Silvana E. Carr et al. (éd.), The Critical Link, Interpreters in the Community, Amsterdam/Philadelphia, John Benjamins, 1997, p. 116.

Stolze, R. (2003): Hermeneutik und Translation, Tübinger Beiträge zur Linguistik 467, Tübingen, Gunter Narr, 348 p.

Cette dernière somme de la pensée herméneutique en traduction, vient s'inscrire dans la ligne de pensée de Stolze, telle qu'elle se manifeste dans de nombreux articles et de façon plus compacte dans Hermeneutisches Übersetzen, également parue chez Narr, en 1992. Onze ans plus tard, Stolze récidive. La similitude des titres pourrait laisser penser que l'on assiste à une réédition plus ou moins corrigée et augmentée. Erreur ! 